\title{
Quiste globulomaxilar de localización inusual: Reporte de un caso
}

\section{An unusual case of globulomaxillary cyst: a case report}

\author{
Orozco Ariza J*, Díaz Caballero A**, Fonseca Ricaurte $M^{* * *}$
}

\section{RESUMEN}

El quiste globulomaxilar es un quiste no odontogénico localizado entre el incisivo lateral y canino del maxilar superior, radiográficamente aparece como una lesión radiolúcida unilocular que desplaza las mencionadas piezas dentarias mostrando una imagen de pera invertida característico de la lesión cuya lámina dura es continua y raras veces interrumpida. La valoración clínica radiográfica es importante para plantear en el diagnóstico diferencial la probable presencia del quiste globulomaxilar. La muestra anatomopatológica debe ser remitida íntegramente para su estudio y diagnóstico definitivo demostrando la presencia de tejido epitelial. Se presenta caso clínico de quiste globulomaxilar en un paciente femenino de 19 años de edad localizado entre canino y primer premolar superior izquierdo.

Palabras clave: Quiste globulomaxilar, quiste fisural, quistes no odontogénicos.

\section{SUMMARY}

Globulomaxillary cyst is a nonodontogenic cyst usually located between maxillary lateral incisor and canine. Radiographic images appears like an unilocular radiolucent lesion that displaced dental teeth showing a invert pear image characteristic of the lesion. X ray and clinical examinations are important to establish the differential diagnosis of globulomaxillary cyst. The hystological examination must be send to its study and definitive diagnoses showing the presence of epithelial tissue. A clinical case of globulomaxillary cyst is presented in a 19 -year- old female patient located between canine and left first premolar maxillary region.

Key words: Globulomaxillary cyst, fissurary cyst, nonodontogenic cyst.

Fecha de recepción: 2 de marzo 2009.

Aceptado para publicación: 6 de marzo 2009.

* Universidad de Cartagena. Facultad de Odontología. Cartagena. Colombia.

** Profesor titular. Universidad de Cartagena. Facultad de Odontología.

*** Odontóloga Universidad de Cartagena. Consultora.

Orozco Ariza J, Díaz Caballero A, Fonseca Ricaurte M. Quiste globulomaxilar de localización inusual: reporte de un caso. Av. Odontoestomatol 2009; 25 (6): 327-330.

\section{INTRODUCCIÓN}

Un quiste es una lesión histológicamente caracterizada por ser una cavidad llena de líquido, células, aire o una combinación de ellos; está rodeado par- cial o totalmente de epitelio y/o tejido conectivo (1). Se generan a partir de la presencia de residuos epiteliales remanentes, que pueden provenir del proceso de fusión de la cara o de la odontogénesis. Los primeros conforman los denominados quistes no odon- 
togénicos o quistes fisurales, los segundos conforman los quistes odontogénicos; de igual forma también pueden aparecer en los maxilares quistes relacionados con una respuesta inflamatoria de lesiones dentales $(1,2)$.

El quiste globulomaxilar incluido en la categoría de quistes no odontogénicos, se forma a partir de restos epiteliales en la región de la fisura incisiva, se localiza entre el incisivo lateral y canino del maxilar superior, suele ser asintomático, aparece sobre todo en la segunda década de la vida, encontrándose una mayor incidencia en mujeres que en hombres con una relación 4:1, al aumentar de tamaño puede causar divergencia en las raíces de los dientes adyacentes y palparse por vestibular. Los dientes vecinos conservan su vitalidad, a menos que se encuentren comprometidos lo cual hace más problemático el diagnóstico diferencial $(3,4)$.

Por lo general el hallazgo se realiza de manera accidental durante el examen radiográfico, mostrando un área radiolúcida unilocular en forma de pera invertida entre las raíces del incisivo lateral y canino, con divergencia en las raíces de estos dientes y cuya lámina dura es continua y raras veces interrumpida (5).

Histológicamente la cavidad quística está revestida por un epitelio escamoso estratificado o cilíndrico ciliado, la pared del quiste muestra infiltración de plasmocitos y linfocitos $(3,5)$.

\section{PRESENTACIÓN DEL CASO}

Paciente de género femenino de 19 años de edad, que acude a la Facultad de Odontología de la Universidad de Cartagena por presentar tumefacción en hemimaxilar izquierdo. Niega antecedentes familiares y sistémicos de importancia en relación con el motivo de consulta. Al examen físico extraoral se evidencia ligera asimetría facial secundaria a tumefacción que compromete región geniana izquierda.

Intraoralmente se observa tumefacción en zona entre órganos dentarios 23 y 24 de aproximadamente 4 centímetros de diámetro con borramiento del surco vestibular y mucosa que recubre con color, textura y humedad dentro de lo normal, de igual forma se evidencia convergencia de las coronas de los órganos dentarios comprometidos, se realizan pruebas de vitalidad pulpar dando resultados positivos. La evaluación radiográfica revela imagen radio lúcida de bordes lisos bien definidos en forma de pera invertida entre órganos dentarios 23 y 24 produciendo desplazamiento de las raíces de los órganos dentarios comprometidos (Fig. 1).

Se realiza un plan de tratamiento bajo anestesia local, técnica a los nervios dentarios posterosuperiores y medios e infraorbitaria lado izquierdo, con refuerzo palatino anterior y posterior, incisión intra surcular con relajante anterior y posterior, ventana ósea y enucleación completa de la membrana quística (Fig. 2) y reposicionamiento de tejidos duros y blandos, finalmente se sutura con seda 4-0.

El procedimiento fue bien tolerado y sin complicaciones, la muestra anatomopatológica se remitió a estudio histopatológico donde confirman la presencia de epitelio escamoso estratificado y quiste maxilar (Fig. 3). Actualmente la paciente tiene 7 años postquirúrgico sin evidencia de recidiva.

\section{DISCUSIÓN}

El quiste globulomaxilar se consideró como un quiste fisural localizado entre los apéndices globular y

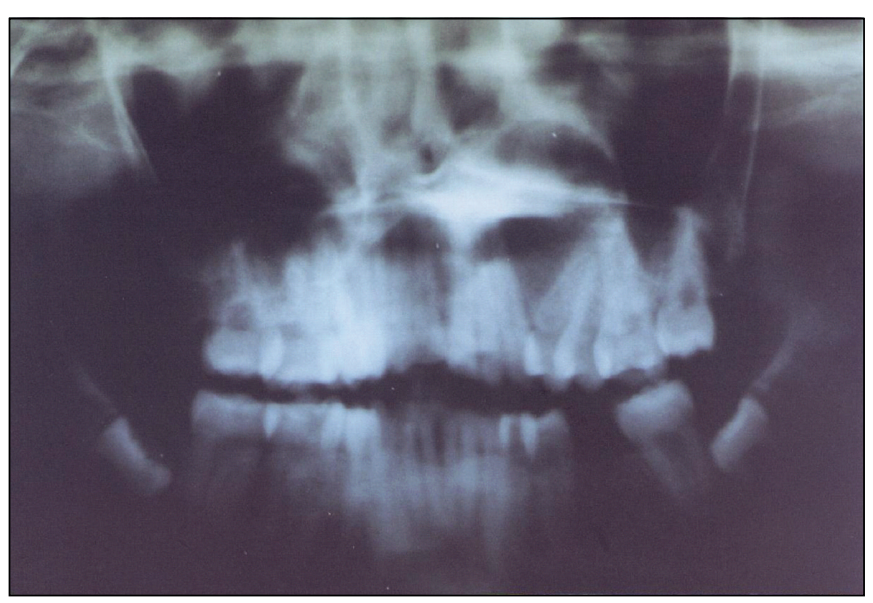

Fig. 1. Radiografía panorámica en la que se observa una amplia imagen radiolúcida en forma de pera invertida entre canino y primer premolar superior izquierdo. 


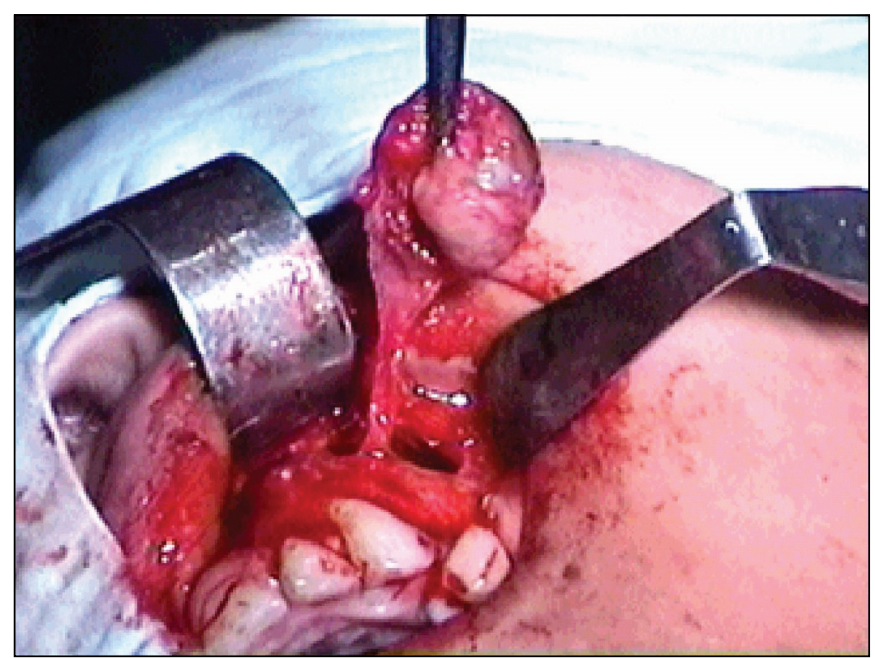

Fig. 2. Enucleación del saco quístico.

maxilar. La antigua teoría sobre el origen de esta lesión relacionaba el atrapamiento de epitelio en una línea de cierre embriológico con la transformación quística subsecuente. En la actualidad, la evidencia muestra que este tipo de quiste se deriva muy probablemente del epitelio odontógeno situado entre el incisivo lateral y canino maxilar (6-8), en el presente caso el quiste se encuentra localizado entre el canino y el primer premolar izquierdo no reportándose en estudios anteriores un caso con igual ubicación.

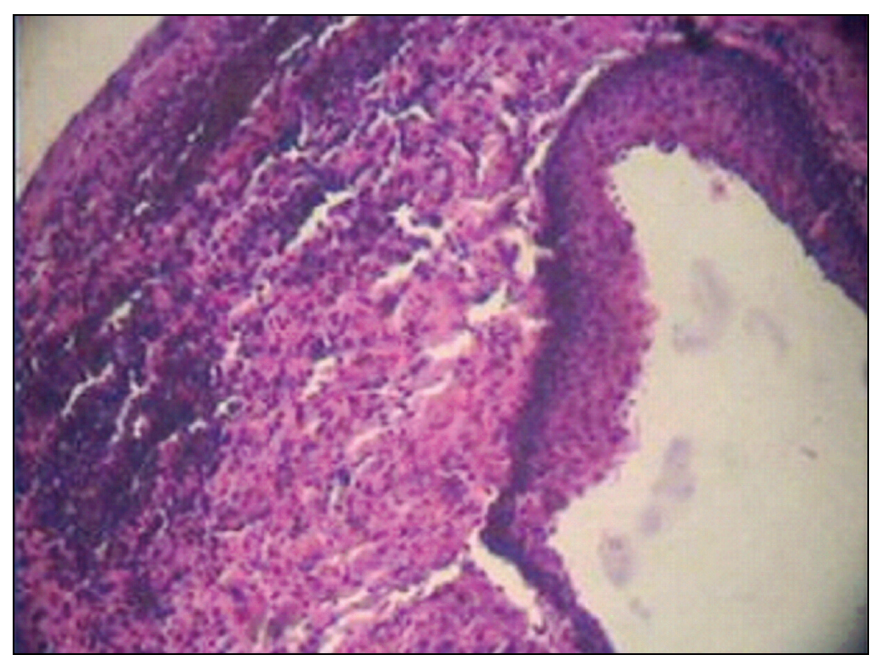

Fig. 3. Estudio histopatológico donde se confirma la presencia de epitelio escamoso estratificado.
La valoración clínica radiográfica meticulosa es importante para plantear en el diagnóstico diferencial la probable presencia del quiste globulomaxilar. Cuando las piezas dentarias adyacentes se encuentran desvitalizadas se hace más complejo aún, con un criterio clínico, el diagnóstico diferencial del quiste globulomaxilar (9).

La muestra anatomopatológica debe ser remitida íntegramente para su estudio y diagnóstico definitivo en el servicio respectivo demostrando la presencia de tejido epitelial (10).

En la actualidad se considera que este quiste podría corresponder a distintas variedades, como el queratoquiste odontogénico, el radicular lateral, o el periodontal lateral. Sin embargo, algunos quistes encontrados en esta localización característica no han sido clasificados todavía en ninguna de las anteriores categorías.

\section{BIBLIOGRAFÍA}

1. Galindo-Moreno PA, Parra-Vázquez MJ, SánchezFernández E, Ávila-Ortiz GA. Maxillary cyst associated with an invaginated tooth: a case report and literature review. Quintessence Int 2003 Jul-Aug; 34(7):509-14.

2. Kramer IRH, Pindborg JJ, Shear M. World Health Organization. International Classification of Tumours. Histological Typing of Odontogenic Tumours (ed 2). Berlin-Budapest: Springer-Verlag; 1992. p. 37.

3. Chimenti C, Monaco A, Nardi E, Volpe F. Globulomaxillary cysts. Minerva Stomatol. 1996 Dec; 45(12):589-92.

4. Gonzalez Perez LM, Moreno Sanchez J. Our experience in the study and treatment of globulomaxillary cysts. Rev Eur Odontoestomatol 1991 Jan-Feb; 3(1):41-8.

5. Abdel-Azim MM. Healing of globulomaxillary cyst after non-surgical endodontic treatment “case report”. Egypt Dent J 1995 Jul; 41(3): 1295-8. 
6. Wysocki GP, Goldblatt LI. The so-called "globulomaxillary cyst" is extinct. Oral Surg Oral Med Oral Pathol 1993 Aug;76(2):185-6.

7. Häring P, Filippi A, Bornstein MM, Altermatt HJ, Buser D, Lambrecht JT. The "globulomaxillary cyst" a specific entity or a myth? Schweiz Monatsschr Zahnmed. 2006;116(4):380-97.

8. Regezi JA, Sciubba JJ. Patología Bucal tercera edición. México: McGraw-Hill Interamericana 2000:310.

9. Steiner DR. A lesion of endodontic origin misdiagnosed as a globulomaxillary cyst. J Endod 1999 Apr;25(4):277-81.
10. Carrasco R, Sancho MA, Cahuana A, Parri FJ, San Vicente B, Morales L. Post-traumatic facial deformity. Globulomaxillary cyst. Cir Pediatr. 1999 Jul;12(3):129-31.

\section{CORRESPONDENCIA}

Antonio Díaz Caballero

Universidad de Cartagena

Facultad de Odontología

Departamento de Investigaciones

Campus de la Salud Barrio Zaragocilla

Cartagena. Colombia

Correo electrónico: antoniodiazc@yahoo.com 